\title{
重复经颖磁刺激治疗阿尔茨海默病患者 的疗效影响因素
}

\author{
王锦涛, 黄 蕾, 魏丽丽, 陈 炜 \\ 浙江大学医学院附属邵逸夫医院精神卫生科, 浙江 杭州 310016
}

\begin{abstract}
[摘要] 重复经颖磁刺激(rTMS) 是一种安全的非侵入性千预技术, 在改善阿尔 茨海默病 $(\mathrm{AD})$ 患者的认知功能上取得了一定的效果, 不同 rTMS 治疗方案之间的疗 效差异可能与其刺激频率、模式、部位、维持时间、强度、聚焦能力等因素有 关。研究表明, 高频 rTMS 的疗效优于低频; 间接 $\theta$ 爆发刺激与常规 rTMS 疗效相当, 但每次治疗时间短, 患者依从性增加; 刺激 AD 患者受损脑区或关联网络可增加疗 效; 短期强化治疗与长期维持治疗结合可维持疗效; 结合认知损害程度动态调整刺 激强度可增加疗效; 利用磁共振功能连接方法等技术可解决 rTMS 的聚焦能力。本 综述分析了上述影响因素, 为今后 $\mathrm{TTMS}$ 治疗 $\mathrm{AD}$ 的临床方案设计提供思路。
\end{abstract}

[关键词] 阿尔茨海默病; 重复经领磁刺激;认知;治疗; 影响因素; 综述

[中图分类号 ］R749.1 [ 文献标志码 ］A

\section{Factors affecting the efficacy of repetitive transcranial magnetic stimulation for patients with Alzheimer's disease}

WANG Jintao, HUANG Lei, WEI Lili, CHEN Wei (Department of Psychiatry, Sir Run Run Shaw Hospital, Zhejiang University School of Medicine, Hangzhou 310016, China) Corresponding author: CHEN Wei, E-mail: srrcw@zju.edu.cn, https://orcid.org/00000003-0903-7945

\begin{abstract}
[ Abstract ] Repetitive transcranial magnetic stimulation (rTMS) is a safe and noninvasive technique. In recent years, many studies have demonstrated that rTMS can improve cognitive function in Alzheimer's disease (AD) patients and has potential as a therapeutic method for AD. However, the efficacy varies greatly with different rTMS treatment regimens, which is related to the frequency, type, location, duration, intensity and focusing power of stimulation. Recent studies have shown that high-frequency stimulation is superior to low-frequency stimulation; efficacy of intermittent theta burst
\end{abstract}

收稿日期: 2021-02-25 接受日期:2021-05-20

基金项目: 国家重点研发计划(2017YFC1310502); 国家自然科学基金(2020C03021); 浙江省重点研发计划(2020C03021) 第一作者: 王锦涛,硕士研究生, 主要从事阿尔茨海默病患者的脑功能研究;E-mail :wangjt@zju.edu.cn; https://orcid.org/ 0000-0002-4649-7492

通信作者: 陈 炜, 主任医师, 硕士生导师, 主要从事认知、情感、睡眠疾病诊治研究;E-mail: srrew@zju.edu.cn; https:// orcid.org/0000-0003-0903-7945 
stimulation (iTBS) is similar to that of conventional rTMS, but iTBS treatment session is shorter and might be more acceptable for AD patients; rTMS stimulation sites targeting AD-damaged brain regions or associated networks would be more effective; short-term intensive treatment combined with long-term maintenance treatment can gain long-term efficacy; dynamic adjustment of stimulus intensity combined with the degree of cognitive impairment can enhance the efficacy; functional connection based on functional magnetic resonance imaging may improve the focusing power of rTMS. In this article, we review the factors related to the efficacy of rTMS, to provide reference for feasible rTMS therapeutic regimens of $\mathrm{AD}$.

[ Key words ] Alzheimer's disease; Repetitive transcranial magnetic stimulation; Cognition; Therapy; Influence factor; Review

[J Zhejiang Univ (Med Sci), 2021, 50(3): 383-389.]

[缩略语］阿尔茨海默病(Alzheimer disease,AD); 经颖磁刺激 (transcranial magnetic stimulation, TMS); 重复经颖磁刺激(repetitive TMS, rTMS); 间接 $\theta$ 爆发刺激(intermittent theta burst stimulation, iTBS); 背外侧前额叶皮层(dorsolateral prefrontal cortex, DLPFC); 静息运动阈值 (rest motor threshold,rMT); 静息态功能磁共振成像 (restingstate functional magnetic resonance imaging,rs-fMRI)

$\mathrm{AD}$ 是一种起病隐袭、进行性发展的神经退 行性疾病, 主要特征为早期出现记忆力下降, 随 着疾病的进展, 逐渐出现两个及以上认知领域的 其他损害, 如执行功能、注意力、语言、社会认知 和判断、心理运动速度、视觉操作或视觉空间能 力, 严重影响个体的日常生活独立性及生存质 量, 给社会、家庭带来沉重的经济和心理负担。 据调查, 我国 AD 患病率约为 $3.94 \%$, 患者人数已

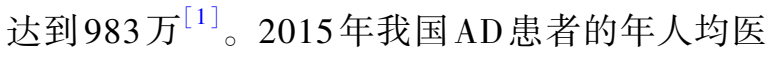
疗费用约为 13 万元, 预计 2030 年总医疗费用将达 到约 17 万亿元 ${ }^{[2]}$ 。临床治疗 $\mathrm{AD}$ 的常用药物为乙 酰胆碱酯酶抑制剂和 N-甲基-D-门冬氨酸受体拮 抗剂。这些药物虽然能在一定程度上改善 $\mathrm{AD}$ 患 者的认知功能和延缓病程, 但疗效有限, 且部分 患者无法耐受 ${ }^{[3]}$ 。针对 $\beta$ 淀粉样蛋白的单克隆抗 体虽能降低 $\mathrm{AD}$ 患者的 $\beta$ 淀粉样蛋白沉积, 但并不 能改善患者的认知功能 ${ }^{[4-5]}$ 。我国最新上市的甘 露特钠胶囊可能通过改变肠道菌群改善 AD 患者 的认知功能, 但还需要进行更多的相关研究以证 实其疗效 ${ }^{[6]}$ 。截至目前, AD 的新型药物开发几乎 都以失败告终, 而传统药物的使用存在诸多限 制 ${ }^{[7]}$ 。因此, 越来越多的研究者开始探索非药物 疗法。
TMS 是一种安全、无创的非侵人性干预技术， 其原理是根据电磁感应效应, 通过在颉外施加由 变化电场产生的变化磁场, 穿过颅骨后在颅内产 生感应电流, 改变神经元膜电位, 从而影响脑内物 质代谢和神经元活动, 引起脑功能变化 ${ }^{[8]}$ 。rTMS 是在一段时间内, 在大脑的特定区域以相同的强 度传递一系列脉冲, 从而对大脑产生一系列刺激, 诱导持久作用, 改变和调节皮层神经元的活动。 因其具有安全、无创等优点, 已广泛应用于抑郁 症、强迫症、物质依赖、脑卒中等各种神经精神疾 病的临床治疗。近年来, rTMS 在 AD 治疗中已取 得了一定疗效, 可改善 AD 患者的认知功能和精神 行为症状等 ${ }^{[9]}$ 。 rTMS 的安全性较好, 常见不良反 应为轻度头痛、耳鸣、短期听力下降、急性精神病 症状等, 癫㾁的发生风险小于 $1 / 1000$, 且是在超出 当前建议高频治疗参数的情况下发生 ${ }^{[10]}$ 。rTMS 治疗 $\mathrm{AD}$ 未见癫㾁等严重不良反应报道, 少数受试 者可能出现轻微头痛。但 $\mathrm{rTMS}$ 在 $\mathrm{AD}$ 治疗中的临 床应用仍未成熟, 各个研究的治疗方案及临床疗 效差异较大, 其治疗效果与刺激频率、模式、部位、 强度、维持时间、聚焦能力等因素有关。本文就上 述影响因素做一综述, 为进一步促进 rTMS未来的 临床应用服务。 


\section{1 rTMS 刺激频率和刺激模式与 AD 的疗效}

rTMS 刺激频率分为高频和低频。高频rTMS (刺激频率至少 $5 \mathrm{~Hz}$ ) 可使目标皮层的兴奋性提 高, 低频 rTMS (刺激频率不超过 $1 \mathrm{~Hz}$ ) 则对目标皮 层的兴奋性具有抑制作用 ${ }^{[11]}$ 。目前, rTMS 在 AD 中的治疗大多选择高频。Ahmed 等 ${ }^{[12]}$ 将 45 例 $\mathrm{AD}$ 患者随机分为 3 组, 分别接受高频 rTMS 刺激 $(20 \mathrm{~Hz})$ 、低频 rTMS 刺激 $(1 \mathrm{~Hz})$ 或假刺激, 结果显 示高频组的工具性日常生活能力量表、简易精神 状态检查和老年抑郁量表的改善程度明显优于 低频组和假刺激组, 且这种改善可维持 3 个月。 荟萃分析结果也显示, 高频rTMS 治疗 AD 患者的

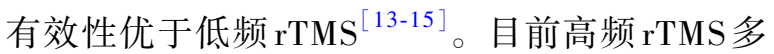
采用 $10 \mathrm{~Hz}$ 或 $20 \mathrm{~Hz}$, 荟萃分析结果显示 $20 \mathrm{~Hz}$ 的 rTMS 具有更好的改善认知功能效果 ${ }^{[15]}$, 但尚无 对这两种刺激频率治疗 AD 患者疗效差异的研 究, 更高频率 (如 $40 \mathrm{~Hz}$ ) 的 rTMS 是否能取得更好 的疗效也需要临床研究来证实。高频 rTMS 可能 可使 $\mathrm{AD}$ 患者的大脑皮层兴奋性提高, 诱导突触 的长时程增强, 以达到认知功能的改善 ${ }^{[16]}$ 。 $A D$ 患者的脑血流量和代谢偏低, 而高频 rTMS 可提 高脑代谢水平和脑血流, 这可能也是高频刺激疗 效较好的原因 ${ }^{[17-18]}$ 。

目前临床常用的 rTMS 刺激模式需要20 $30 \min ^{[19]}$, 这可能是影响 AD 患者疗效的一个因 素。 $\mathrm{AD}$ 患者的配合程度比较差, 很难做到长时间 保持头部位置固定, 而头部活动会导致 rTMS 刺激 靶点的移动。iTBS 是 rTMS 的一种特殊模式, 其每 次治疗只需几分钟, 可减少靶点移动带来的误 差 ${ }^{[20]}$ 。Wu 等 $^{[21]}$ 发现对左侧DLPFC作iTBS 能显著 改善 AD 患者的认知功能尤其是记忆功能。一项 抑有症的多中心研究发现 iTBS 与常规 rTMS 疗效 相当 ${ }^{[22]}$, 但iTBS 的费用约为常规 rTMS 的三分之 二 ${ }^{[23]}$, 目前无 AD 患者的相关研究。iTBS 具有治 疗时间短、费用低的优势, 今后应开展更多大样 本、多中心 iTBS 治疗 AD 研究以明确其疗效。

\section{2 rTMS 刺激部位与 AD 的疗效}

rTMS 的作用方式有以下三种: 直接靶向、距 离效应和分布式调制。大多数 rTMS研究都基于 其直接靶向作用, 即直接刺激某个功能障碍脑区, 使该大脑区域受损的神经元活动得以调节, 改善
其所支持的功能; 距离效应是 rTMS 刺激与功能障 碍脑区互相关联的神经区域,通过该区域与功能 障碍脑区的相互作用来调节功能障碍脑区受损的 神经元活动; 分布式调制则是通过刺激诱导特定 网络的神经递质释放来调节大脑活动, 从而使异 常活动的网络趋于正常 ${ }^{[24]}$ 。因此,刺激不同脑区 可能带来不同的效果。早期的研究主要刺激 $\mathrm{DLPFC}_{0}$ 。Cotelli等 ${ }^{[25-26]}$ 在 $\mathrm{AD}$ 患者执行物体和动 作图画命名任务过程中对其左侧或右侧 DLPFC 给 予 $20 \mathrm{~Hz}$ 的rTMS, 发现 rTMS 能提高 AD 患者的命名 准确性, 改善认知功能。进一步研究发现, 与假刺 激相比, $20 \mathrm{~Hz}$ 的 rTMS 刺激左侧 DLPFC 两周可显 著改善患者的听觉理解成绩, 且这种效果可保持 8 周左右 ${ }^{[27]}$ 。Padala 等 ${ }^{[28]}$ 发现 $10 \mathrm{~Hz}$ 的 rTMS 刺激左 侧 DLPFC 四周可改善 AD 患者的淡漠症状和认知 功能, 且对淡漠症状的改善持续至治疗结束后 12 周。一项纳人 13 个随机对照试验共 293 例 AD 患 者的荟萃分析结果显示, 左侧和右侧 DLPFC 的高 频rTMS 均可改善AD 患者的认知功能 ${ }^{[29]}$ 。

除了DLPFC, 研究者也探索了刺激其他脑区 对 $\mathrm{AD}$ 患者的影响。用 $10 \mathrm{~Hz}$ 的 rTMS 刺激右额下回 和右颢上回, 发现刺激右额下回能改善 $\mathrm{AD}$ 患者的 注意力、精神运动速度和执行功能 ${ }^{[30]}$ 。Koch 等 ${ }^{[31]}$ 发现,rTMS 刺激 AD 患者的楔前叶可以特异性地 改善情景记忆功能; 脑电图显示,rTMS 治疗后患者 楔前叶的神经活动增加, $\beta$ 波段的脑部振荡增强, 楔前叶与默认网络内侧额叶之间的功能连接增 强。Alcala-Lozano 等 ${ }^{[32]}$ 将刺激左侧 DLPFC 与刺激 六个脑区 (Broca区、Wernicke区、双侧DLPFC、双 侧顶叶躯体感觉联合皮层)进行比较,发现疗效无 差异。Cheng 等 ${ }^{[14]}$ 的荟萃分析 (8 项研究, 194 例患 者)结果也显示多靶点与单靶点的疗效无差异。 但 Lin 等 ${ }^{[33]}$ 最近进行的一项荟萃分析 (12 项研究, 231 例患者) 结果显示, 多靶点刺激比单靶点治疗 效果好。今后需要开展大样本、多中心研究来 验证。

综上, DLPFC 是最先、也是在 $\mathrm{AD}$ 患者中实施 rTMS 刺激最多的脑区,rTMS不仅可改善DLPFC 的 局部脑活动, 而且可改善其关联深部脑区或脑 网络的神经活动, 从而改善 $\mathrm{AD}$ 患者的认知功 能 ${ }^{[34-35]}$ 。这一推测也在楔前叶中得到验证。 Koch 等 ${ }^{[31]}$ 发现楔前叶处rTMS 可能通过改善其局 部和所在默认网络的神经活动, 从而改善 $\mathrm{AD}$ 患者 
的记忆功能。 $\mathrm{AD}$ 是一种多脑区、多脑网络、多种 认知领域受损的神经退行性疾病 ${ }^{[36]}$, rTMS 刺激多 个脑区可能具有较好的疗效, 但由于rTMS 的距离 效应和网络效应,必须考虑多个脑区之间的相互 作用, 以免产生拮抗作用。盲目刺激多个脑区,一 方面可能会导致不同脑区之间的刺激作用互相拮 抗, 另一方面也可能会刺激无效脑区。因此针对 $\mathrm{AD}$ 受损脑区或关联网络, 寻找最有效刺激点进行 rTMS 刺激的方案可能更合适。

\section{3 rTMS 刺激维持时间、刺激强度与 AD 的疗效}

rTMS 刺激效应的维持时间与刺激时长成正 比, 即刺激时间越长, 刺激效应的维持时间越 长 $^{[11]}$ 。荟萃分析结果显示, 长期 $\mathrm{rTMS}$ (超过 5 次) 比短期 $\mathrm{rTMS}$ (少于 3 次) 对 AD 患者认知功能的改 善有更好的作用 ${ }^{[33]}$ 。Cotelli 等 ${ }^{[27]}$ 发现 10 次和 20 次左侧 DLPFC 的 rTMS 均可改善AD 患者的听觉理 解成绩, 且均可保持 8 周左右。最近一项纳人 75 例患者的研究显示, $20 \mathrm{~Hz}$ 左侧 DLPFC 的rTMS 治 疗 6 周 (5 次/周)后, $\mathrm{AD}$ 患者的认知功能改善, 其中 简易精神状态检查评分的改善可维持 12 周左右, 皮质可塑性的提高与 rTMS 治疗 AD 患者的认知改 善相关 ${ }^{[37]}$ 。研究者对 AD 患者先进行 6 周 rTMS 结 合认知训练的强化治疗 (5 次/周), 再进行 3 个月 的维持治疗 (2 次/周), 发现患者认知功能均有显 著改善 ${ }^{[38-39]}$ 。rTMS 刺激效应的维持时间与刺激 时长成正比的原因可能与长时程增强有关, 刺激 时长越久, 长时程增强效应更强更持久 ${ }^{[40]}$ 。虽然 有研究显示 10 次和 20 次 rTMS 的疗效维持时间相 当,但较长的刺激时间一般会使疗效维持时间更 久。因此, 先进行短时间的 rTMS 强化治疗以快速 取得治疗效果, 后进行长期 rTMS 维持治疗以维持 疗效可能成为未来的趋势。

在刺激强度方面,临床采用静息状态时 TMS 作用于运动皮层引起所支配肌肉收缩的最小强 度, 即 $\mathrm{rMT}$, 通常以 $80 \%$ 120\% rMT 的某一个强度 给患者治疗。健康受试者给予 $50 \% 、 75 \% 、 100 \%$ 的 rMT 刺激,其N-back 任务的认知成绩提高, 反应时 间缩短, 其中以 $75 \%$ 的 $\mathrm{rMT}$ 最显著 ${ }^{[41]}$ 。另一项研 究采用 $60 \% 、 80 \% 、 100 \% 、 120 \%$ 的 $\mathrm{rMT}$ 刺激,发现 脑皮层的兴奋性与刺激强度呈依赖效应 ${ }^{[42]}$ 。在 $\mathrm{AD}$ 患者中, 不同皮质之间的功能连接通常遭到破 坏。有研究显示, $90 \%$ rMT 的 TMS 刺激健康受试
者的顶叶能提高其运动皮层的兴奋性, 但在 $\mathrm{AD}$ 患 者中需要 $110 \%$ 的 $\mathrm{rMT}$ 强度, 而且运动皮层兴奋性 与认知功能表现成正比, 提示认知性能较好患者 的功能连接受损较小, 所需的 rMT 强度较小 ${ }^{[43]}$ 。

因此, 最佳rTMS 刺激强度可能与患者的认知功能 有关, 今后应进一步明确不同刺激强度与 rTMS 治 疗 $\mathrm{AD}$ 疗效的关系,开发动态调整刺激强度治疗不 同认知功能损害 $\mathrm{AD}$ 的方法。

\section{4 rTMS 聚焦能力与 AD 的疗效}

海马是参与情景记忆形成的重要脑部结构, 同时也参与其他认知功能的产生, 是 $\mathrm{AD}$ 患者最先 产生病变的脑区 ${ }^{[44-46]}$ 。要提高 $\mathrm{AD}$ 的治疗效果, 直 接或间接刺激海马非常关键。研究发现, 通过植 人的深部脑电极直接刺激海马体可显著改善记忆 功能 ${ }^{[47-48]}$, 但此方法费用高、有创, 并不适合 $\mathrm{AD}$ 患 者。由于海马位于大脑深处, 而常用的 rTMS 治疗 仪是 “8”字线圈, 其聚焦点在 $3 \mathrm{~cm}$ 或更浅, 只能 聚焦于大脑皮层 $(2 \mathrm{~cm})$, 无法刺激深部脑组织; 深 部TMS 刺激可达 $6 \mathrm{~cm}$, 可解决聚焦深度的问题, 且 不损伤浅层皮质,高效刺激大脑深部核团,但目前 深部TMS 的刺激线圈为 $\mathrm{H}$ 型, 只能刺激脑岛等深部 脑区, 仍无法刺激海马 ${ }^{[49-51]}$ 。

为了克服这一困难, Wang 等 ${ }^{[52]}$ 发明了一种 间接的刺激方法, 即通过刺激网络中的节点, 将该 节点的刺激效应传输到网络内的其他节点。基于 皮质-海马网络, 研究者在 rs-fMRI引导下, 使用高 频rTMS 个体化刺激顶叶皮层来干预海马的神经 活动, 发现健康受试者经 rTMS 刺激后不但记忆功 能显著提高, 而且海马及其网络的神经活动也得 到了显著增强。这一研究方法和结论已经在健康 受试者或认知损伤患者中得到了验证 ${ }^{[53-58]}$ 。 Velioglu 等 ${ }^{[59]}$ 发现, 个体化刺激顶叶的海马靶向 rTMS 可以提高 AD 患者的视觉识别记忆功能和时 钟绘制测试分数。本研究团队初步研究发现, 在 rs-fMRI引导下个体化刺激顶叶的海马靶向 rTMS 可以改善 $\mathrm{AD}$ 患者的记忆和整体认知功能 ${ }^{[60]}$, 并 且这种改善与海马脑血流量、默认网络内功能连 接强度增加相关, 提示 rTMS 可调节海马神经活动 和默认网络内功能连接, 从而改善 $\mathrm{AD}$ 患者记忆功 能和整体认知功能(待发表)。针对 $\mathrm{AD}$ 受损脑区 或关联网络进行功能连接定位的 rTMS 刺激可能 成为未来的趋势。 


\section{5 结 语}

rTMS 作为 $\mathrm{AD}$ 的非药物治疗方法, 在改善 $\mathrm{AD}$ 患者认知功能、记忆等方面已取得较好的效果。 已有研究表明,频率为 $10 \sim 20 \mathrm{~Hz}$ 、强度为 $90 \%$ $120 \%$ rMT 的 rTMS 刺激 AD 患者 DLPFC、楔前叶、 左顶叶等脑区 1 6 周, 能改善患者的认知功能, 且 疗效能维持一段时间; 高频疗效优于低频, 可进一 步探索更高频率(如 $40 \mathrm{~Hz}$ ) 的 rTMS 疗效; iTBS 具有 治疗时间短、费用低等优势, 可以进一步研究其与 rTMS 的疗效差异; 持续较长的刺激时间会使疗效 维持时间更久, 随着 rTMS 治疗仪器逐步家庭化, 短期强化治疗结合长期维持治疗的方案具有广阔 的前景; 最佳刺激强度与患者的认知功能有关, 今 后可根据患者认知损害程度的变化动态调整刺激 强度, 并且可基于磁共振功能连接方法等技术解 决 rTMS 的聚焦能力, 针对 AD 受损脑区或关联网 络进行个体化的有效刺激。

建议今后聚焦下列问题: (1) rTMS 治疗 AD 的 效果主要取决于刺激靶点、频率、模式、强度、治疗 时间等因素, 如何理清这些因素与疗效的关系, 解 决靶点定位技术手段模糊、定位准确性差等关键问 题,利用磁共振导航等技术精确定位, 提高 rTMS临 床疗效。(2)目前临床研究样本量小, 随机双盲试验 少, 普遍缺乏多中心、长期跟踪研究, 今后应开展多 中心、大样本、前瞻性研究, 提高 rTMS 治疗 AD疗效 的证据等级。3rTMS 存在聚焦能力不足, 常用的 “8” 字线圈及 $\mathrm{H}$ 型线圈无法满足 $\mathrm{AD}$ 治疗的需要, 须进一步研发与深部TMS 配套的线圈、基于磁共振 功能连接方法等技术。(4)从动物模型、尸体解剖及 临床各方面采用分子生物学、细胞生物学、影像学、 电生理等相关技术, 阐明 rTMS 治疗 AD 的相关机 制。(5)建立实时、动态的个体化治疗技术和方案。 由于不同 $\mathrm{AD}$ 患者脑皮层活动各不相同, 疾病的严 重程度、临床表现、共病躯体疾病、遗传等因素各不 相同, 临床疗效差异显著, 应对相关因素进行综合 分析,通过人工智能等信息技术建立模型, 实施个 体化治疗方案。相信随着基础研究和临床应用的 不断深人,rTMS 将为 $\mathrm{AD}$ 患者带来福音。

利益冲突 所有作者均声明不存在利益冲突

\section{参考文献}

[1] JIA L, DU Y, CHU L, et al. Prevalence, risk factors, and management of dementia and mild cognitive impairment in adults aged 60 years or older in China: a cross-sectional study $[\mathrm{J} / \mathrm{OL}]$. Lancet Public Health, 2020, 5(12): e661-e671.

[2] JIA J, WEI C, CHEN S, et al. The cost of Alzheimer's disease in China and re-estimation of costs worldwide $[J]$. Alzheimers Dement, 2018, 14(4): 483-491.

[3] KRANTIC S. Editorial: from current diagnostic tools and therapeutics for Alzheimer's disease towards earlier diagnostic markers and treatment targets $[\mathrm{J}]$. Curr Alzheimer Res, 2017, 14(1): 2-5.

[4] VANDENBERGHE R, RINNE J O, BOADA M, et al. Bapineuzumab for mild to moderate Alzheimer's disease in two global, randomized, phase 3 trials $[\mathrm{J}]$. Alzheimers Res Ther, 2016, 8(1): 18.

[5] KITAMURA S, NAKAMURA Y, HOMMA A, et al. Tolerability and efficacy of the long-term administration of memantine hydrochloride (Memary $\left.{ }^{\circledR}\right)$ in patients with moderate to severe Alzheimer's disease $[\mathrm{J}]$. Nippon Ronen Igakkai Zasshi, 2014, 51(1): 74-84.

[6] XIAO S, CHAN P, WANG T, et al. A 36-week multicenter, randomized, double-blind, placebo-controlled, parallel-group, phase 3 clinical trial of sodium oligomannate for mild-to-moderate Alzheimer's dementia[J]. Alzheimers Res Ther, 2021, 13(1): 62.

[7] CUMMINGS J, LEE G, RITTER A, et al. Alzheimer's disease drug development pipeline: 2019[J]. Alzheimers Dement, 2019, 5(1): 272-293.

[8] WASSERMANN E M, LISANBY S H. Therapeutic application of repetitive transcranial magnetic stimulation: a review[J]. Clin NeuroPhysiol, 2001, 112(8): 1367-1377.

[9] LEFAUCHEUR J P, ALEMAN A, BAEKEN C, et al. Evidence-based guidelines on the therapeutic use of repetitive transcranial magnetic stimulation (rTMS): an update (2014-2018)[J]. Clin NeuroPhysiol, 2020, 131(2): 474-528.

[10] GONSALVEZ I, BAROR R, FRIED P, et al. Therapeutic noninvasive brain stimulation in Alzheimer's disease [J]. Curr Alzheimer Res, 2017, 14(4): 362376.

[11] KLOMJAI W, KATZ R, LACKMY-VALLÉE A. Basic principles of transcranial magnetic stimulation (TMS) and repetitive TMS (rTMS) $[\mathrm{J}]$. Ann Phys Rehabil Med, 2015, 58(4): 208-213.

[12] AHMED M A, DARWISH E S, KHEDR E M, et al. Effects of low versus high frequencies of repetitive transcranial magnetic stimulation on cognitive function and cortical excitability in Alzheimer's dementia[J]. J Neurol, 2012, 259(1): 83-92.

[13 ] LIAO X, LI G, WANG A, et al. Repetitive transcranial magnetic stimulation as an alternative therapy for cognitive impairment in Alzheimer's disease: a metaanalysis[J]. J Alzheimer Dis, 2015, 48(2): 463-472.

[14 ] CHENG C P W, WONG C S M, LEE K K, et al. Effects of repetitive transcranial magnetic stimulation on 
improvement of cognition in elderly patients with cognitive impairment: a systematic review and metaanalysis $[\mathrm{J} / \mathrm{OL}]$. Int J Geriatr Psychiatry, 2018, 33(1): e1-e13.

[15] WANG X, MAO Z, LING Z, et al. Repetitive transcranial magnetic stimulation for cognitive impairment in Alzheimer's disease: a meta-analysis of randomized controlled trials $[\mathrm{J}]$. J Neurol, 2020, 267(3): 791-801.

[16] ESSER S K, HUBER R, MASSIMINI M, et al. A direct demonstration of cortical LTP in humans: a combined TMS/EEG study $[\mathrm{J}]$. Brain Res Bull, 2006, 69(1): 86-94.

[17] AUSTIN B P, NAIR V A, MEIER T B, et al. Effects of hypoperfusion in Alzheimer's disease[J]. J Alzheimer Dis, 2011, 26 Suppl 3: 123-133.

[18] GUSE B, FALKAI P, WOBROCK T. Cognitive effects of high-frequency repetitive transcranial magnetic stimulation: a systematic review $[\mathrm{J}]$. J Neural Transm, 2010, 117(1): 105-122.

[19] LEFAUCHEUR J P, ANDRÉ-OBADIA N, ANTAL A, et al. Evidence-based guidelines on the therapeutic use of repetitive transcranial magnetic stimulation (rTMS)[J]. Clin NeuroPhysiol, 2014, 125(11): 21502206.

[20] HUANG Y Z, EDWARDS M J, ROUNIS E, et al. Theta burst stimulation of the human motor cortex $[\mathrm{J}]$. Neuron, 2005, 45(2): 201-206.

[21] WU X, JI G J, GENG Z, et al. Strengthened theta-burst transcranial magnetic stimulation as an adjunctive treatment for Alzheimer's disease: an open-label pilot study [J]. Brain Stimulation, 2020, 13(2): 484-486.

[22] BLUMBERGER D M, VILA-RODRIGUEZ F, THORPE K E, et al. Effectiveness of theta burst versus high-frequency repetitive transcranial magnetic stimulation in patients with depression (THREE-D): a randomised non-inferiority trial $[\mathrm{J}]$. Lancet, 2018, 391(10131): 1683-1692.

[23] MENDLOWITZ A B, SHANBOUR A, DOWNAR J, et al. Implementation of intermittent theta burst stimulation compared to conventional repetitive transcranial magnetic stimulation in patients with treatment resistant depression: a cost analysis $[\mathrm{J} / \mathrm{OL}]$. PLoS One, 2019, 14(9): e0222546.

[24] ROTENBERG A, HORVATH J C, PASCUAL-LEONE A. Transcranial magnetic stimulation $[M]$. New York: Springer, 2014: 235-257.

[25] COTELLI M, MANENTI R, CAPPA S F, et al. Effect of transcranial magnetic stimulation on action naming in patients with Alzheimer disease[J]. Arch Neurol, 2006, 63(11): 1602-1604.

[26] COTELLI M, MANENTI R, CAPPA S F, et al. Transcranial magnetic stimulation improves naming in Alzheimer disease patients at different stages of cognitive decline $[J]$. Eur J Neurol, 2008, 15(12): 1286-1292.
[27] COTELLI M, CALABRIA M, MANENTI R, et al. Improved language performance in Alzheimer disease following brain stimulation $[\mathrm{J}]$. J Neurol Neurosurg Psychiatry, 2011, 82(7): 794-797.

[28] PADALA P R, BOOZER E M, LENSING S Y, et al. Neuromodulation for apathy in Alzheimer's disease: a double-blind, randomized, sham-controlled pilot study[J]. J Alzheimer Dis, 2020, 77(4): 1483-1493.

[29] CHOU Y H, TON THAT V, SUNDMAN M. A systematic review and meta-analysis of rTMS effects on cognitive enhancement in mild cognitive impairment and Alzheimer's disease $[\mathrm{J}]$. NeuroBiol Aging, 2020, 86: 1-10.

[30] ELIASOVA I, ANDERKOVA L, MARECEK R, et al. Non-invasive brain stimulation of the right inferior frontal gyrus may improve attention in early Alzheimer's disease: a pilot study $[\mathrm{J}]$. J Neurological Sci, 2014, 346(1-2): 318-322.

[31] KOCH G, BONNİ S, PELLICCIARI M C, et al. Transcranial magnetic stimulation of the precuneus enhances memory and neural activity in prodromal Alzheimer's disease $[\mathrm{J}]$. NeuroImage, 2018, 169: 302-311.

[32] ALCALÁ-LOZANO R, MORELOS-SANTANA E, CORTÉS-SOTRES J F, et al. Similar clinical improvement and maintenance after rTMS at $5 \mathrm{~Hz}$ using a simple vs. complex protocol in Alzheimer's disease $[\mathrm{J}]$. Brain Stimul, 2018, 11(3): 625-627.

[33] LIN Y, JIANG W J, SHAN P Y, et al. The role of repetitive transcranial magnetic stimulation (rTMS) in the treatment of cognitive impairment in patients with Alzheimer's disease: a systematic review and metaanalysis[J]. J Neurol Sci, 2019, 398: 184-191.

[34] NARDONE R, SEBASTIANELLI L, VERSACE V, et al. TMS-EEG co-registration in patients with mild cognitive impairment, Alzheimer's disease and other dementias: a systematic review [J]. Brain Sci, 2021, 11(3): 303.

[35] IIMORI T, NAKAJIMA S, MIYAZAKI T, et al. Effectiveness of the prefrontal repetitive transcranial magnetic stimulation on cognitive profiles in depression, schizophrenia, and Alzheimer's disease: a systematic review $[\mathrm{J}]$. Prog Neuro-PsychoPharmacol Biol Psychiatry, 2019, 88: 31-40.

[36] ZHOU J, SEELEY W W. Network dysfunction in Alzheimer's disease and frontotemporal dementia: implications for psychiatry $[\mathrm{J}]$. Biol Psychiatry, 2014, 75(7): 565-573.

[37] LI X, QI G, YU C, et al. Cortical plasticity is correlated with cognitive improvement in Alzheimer's disease patients after rTMS treatment $[J]$. Brain Stimul, 2021, 14(3): 503-510.

[38] BENTWICH J, DOBRONEVSKY E, AICHENBAUM $\mathrm{S}$, et al. Beneficial effect of repetitive transcranial magnetic stimulation combined with cognitive training for the treatment of Alzheimer's disease: a proof of 
concept study $[$ J $]$. J Neural Transm, 2011, 118(3): 463-471.

[39] RABEY J M, DOBRONEVSKY E, AICHENBAUM S, et al. Repetitive transcranial magnetic stimulation combined with cognitive training is a safe and effective modality for the treatment of Alzheimer's disease: a randomized, double-blind study $[\mathrm{J}]$. J Neural Transm, 2013, 120(5): 813-819.

[40] LUBER B, LISANBY S H. Enhancement of human cognitive performance using transcranial magnetic stimulation (TMS) [J]. NeuroImage, 2014, 85: 961970.

[41] CHUNG S W, ROGASCH N C, HOY K E, et al. Impact of different intensities of intermittent theta burst stimulation on the cortical properties during TMSEEG and working memory performance $[\mathrm{J}]$. Hum Brain Mapp, 2018, 39(2): 783-802.

[42] KÄHKÖNEN S, KOMSSI S, WILENIUS J, et al. Prefrontal transcranial magnetic stimulation produces intensity-dependent EEG responses in humans $[\mathrm{J}]$. NeuroImage, 2005, 24(4): 955-960.

[43] BONNİ S, LUPO F, LO GERFO E, et al. Altered parietal-motor connections in Alzheimer's disease patients[J]. J Alzheimer Dis, 2012, 33(2): 525-533.

[44] PRESTON A R, EICHENBAUM H. Interplay of hippocampus and prefrontal cortex in memory $[\mathrm{J}]$. Curr Biol, 2013, 23(17): R764-R773.

[45] OLSEN R K, MOSES S N, RIGGS L, et al. The hippocampus supports multiple cognitive processes through relational binding and comparison $[\mathrm{J}]$. Front Hum Neurosci, 2012, 6: 146.

[46] BALLARD C, GAUTHIER S, CORBETT A et al. Alzheimer's disease [J]. Lancet, 2011, 377: 10191031.

[47] SUTHANA N, HANEEF Z, STERN J, et al. Memory enhancement and deep-brain stimulation of the entorhinal area $[J]$. N Engl J Med, 2012, 366(6): 502-510.

[48] JUN S, KIM J S, CHUNG C K. Direct stimulation of human hippocampus during verbal associative encoding enhances subsequent memory recollection $[\mathrm{J}]$. Front Hum Neurosci, 2019, 13: 23.

[49] ROTH Y, AMIR A, LEVKOVITZ Y, et al. Threedimensional distribution of the electric field induced in the brain by transcranial magnetic stimulation using figure-8 and deep H-coils[J]. J Clin NeuroPhysiol, 2007, 24(1): 31-38.

[50] ROTH Y, PELL G S, CHISTYAKOV A V, et al. Motor cortex activation by $\mathrm{H}$-coil and figure- 8 coil at different depths. Combined motor threshold and electric field distribution study $[\mathrm{J}]$. Clin NeuroPhysiol, 2014, 125(2): 336-343.
[51] LU M, UENO S. Comparison of the induced fields using different coil configurations during deep transcranial magnetic stimulation $[\mathrm{J} / \mathrm{OL}]$. PLoS One, 2017, 12(6): e0178422.

[52] WANG J X, ROGERS L M, GROSS E Z, et al. Targeted enhancement of cortical-hippocampal brain networks and associative memory [J]. Science, 2014, 345(6200): 1054-1057.

[53 ] WANG H, JIN J, CUI D, et al. Cortico-hippocampal brain connectivity-guided repetitive transcranial magnetic stimulation enhances face-cued word-based associative memory in the short term $[\mathrm{J}]$. Front Hum Neurosci, 2020, 14: 541791.

[54] CUI X, REN W, ZHENG Z, et al. Repetitive transcranial magnetic stimulation improved source memory and modulated recollection-based retrieval in healthy older adults $[\mathrm{J}]$. Front Psychol, 2020, 11: 1137.

[55] FREEDBERG M, REEVES J A, TOADER A C, et al. Optimizing hippocampal-cortical network modulation via repetitive transcranial magnetic stimulation: a dose-finding study using the continual reassessment $\operatorname{method}[\mathrm{J}]$. Neuromodulation, 2020, 23(3): 366-372.

[56] FREEDBERG M, REEVES J A, TOADER A C, et al. Persistent enhancement of hippocampal network connectivity by parietal rtms is reproducible $[\mathrm{J}]$. eNeuro, 2019, 6(5): ENEURO.0129-19.2019

[57] HERMILLER M S, KARP E, NILAKANTAN A S, et al. Episodic memory improvements due to noninvasive stimulation targeting the cortical-hippocampal network: a replication and extension experiment $[\mathrm{J} / \mathrm{OL}]$. Brain Behav, 2019, 9(12): e01393.

[58 ] TAYLOR J L, HAMBRO B C, STROSSMAN N D, et al. The effects of repetitive transcranial magnetic stimulation in older adults with mild cognitive impairment: a protocol for a randomized, controlled three-arm trial $[J]$. BMC Neurol, 2019, 19(1): 326.

[59] VELIOGLU H A, HANOGLU L, BAYRAKTAROGLU $\mathrm{Z}$, et al. Left lateral parietal rTMS improves cognition and modulates resting brain connectivity in patients with Alzheimer's disease: possible role of BDNF and oxidative stress $[\mathrm{J}]$. NeuroBiol Learn Mem, 2021, 180: 107410 .

[60] JIA Y, XU L, YANG K, et al. Precision repetitive transcranial magnetic stimulation over the left parietal cortex improves memory in Alzheimer's disease: a randomized, double-blind, sham-controlled study $[\mathrm{J}]$. Front Aging Neurosci, 2021, 13: 693611.

$$
\text { [本文编辑 李 璐 余 方] }
$$

